\title{
Structure of the $\mathrm{RNiO}_{3}$ single crystals $(R=\mathrm{Nd}, \mathrm{Sm}, \mathrm{Gd}, \mathrm{Dy}, \mathrm{Y}, \mathrm{Ho}, \mathrm{Er}, \mathrm{Lu})$
}

\author{
D. J. Gawryluk¹, Y. M. Klein ${ }^{1}$, M. Kozłowski ${ }^{2}$ Ph. Lacorre ${ }^{3}$, A. Linden ${ }^{4}$, M. Medarde ${ }^{1}$ \\ ${ }^{1}$ Laboratory for Multiscale Materials Experiments, Paul-Scherrer-Institut, Forschungsstrasse 111, 5232 Villigen PSI, Switzerland, \\ ${ }^{2}$ Eukasiewicz Research Network Tele \& Radio Research Institute, 11 Ratusznowa Street, 03-450 Warsaw, Poland, \\ ${ }^{3}$ Institut des Molécules et Materiaux du Mans (IMMM) - UMR 6283 CNRS, Le Mans Université, \\ Avenue Olivier Messiaen, 72085 Le Mans, France, \\ ${ }^{4}$ Department of Chemistry, University of Zürich, Winterthurerstrasse 190, 8057 Zürich, Switzerland
}

dariusz.gawryluk@psi.ch

This Stoichiometric rare earth nickelates $\left(R \mathrm{NiO}_{3}\right)$ are a textbook example of strongly correlated electron materials, which provide a notable opportunity to study the interplay between, lattice, charge, and spin degrees of freedom. Their most remarkable characteristic is the presence of spontaneous, temperature-driven metal-to-insulator transitions (MITs) at temperatures $T_{M I T}$ that increase for smaller $R$ ionic radii [1]. Since this happens in absence of Ni mixed valence or chemical disorder, nickelates are perfect, extremely clean model systems for the investigation of the boundary between localized and itinerant behaviour in theoretical studies. Although the existence of the MIT in $R \mathrm{NiO}_{3}$ has been known since 1991 [2], the mechanism(s) at the origin of the spontaneous electronic localization is still the subject of lively debate. In particular, it is unclear how electronic correlations, lattice, and magnetic degrees of freedom interact and lead to the gap opening. Moreover, other interesting phenomena such as an unusual non-centrosymmetric antiferromagnetic ordering [3], superconductivity [4,5] or multiferroelectricity [6, 7] have been either observed or theoretically predicted.

An important drawback for the advancement in the comprehension of the complex nickelate physics has been the limited amount of experimental information, needed to validate theoretical predictions. The reason behind is their challenging chemistry, which requires the use of high oxygen pressure and high temperature during synthesis. These extreme conditions have prevented to date the growth of sizable bulk single crystals with $R \neq \mathrm{La}$, $\mathrm{Pr}$ and $\mathrm{Nd}$. Here we present the first successful growth of $R \mathrm{NiO}_{3}$ single crystals with $R=\mathrm{Nd}$, $\mathrm{Sm}, \mathrm{Gd}$, Dy, Y, Ho, Er, and Lu with sizes up to $\sim 100 \mu \mathrm{m}$, achieved by applying the solvothermal method in temperature gradient under 2000 bar oxygen pressure [8]. We also report a detailed structural and physical property characterization illustrating the excellent quality of the obtained bulk $\mathrm{RNiO}_{3}$ crystals, long time considered impossible to growth.

[1] Gawryluk, D. J. Klein, Y. M. Shang, T. Sheptyakov, D. Keller, L. Casati, N. Lacorre, Ph. Fernández-Díaz, M. T. Rodríguez-Carvajal, J. Medarde, M. (2019). Phys. Rev. B 100, 205137.

[2] Lacorre, Ph. Torrance, J.B. Pannetier, J. Nazzal, A.I. Wang, P.W. Huang T.C. (1991). J. Solid State Chem. $91,225$.

[3] Alonso, J. A. García-Muñoz, J. L. Fernández-Díaz, M. T. Aranda, M. A. G. Martínez-Lope, M. J. Casais, M. T. (1999). Phys. Rev. Lett. 82, 3871.

[4] Chaloupka, J. Khaliullin, G. (2008). Phys. Rev. Lett. 100, 016404.

[5] Li, D. Lee, K. Wang, B. Y. Osada, M. Crossley, S. Lee, H. R. Cui, Y. Hikita, Y. Hwang, H. Y. (2019). Nature $572,624$.

[6] Giovannetti, G. Kumar, S. Khomskii, D. Picozzi, S. van den Brink, J. (2009). Phys. Rev. Lett. 103, 156401.

[7] Perez-Mato, J. M. Gallego, S. V. Elcoro, L. Tasci, E Aroyo, M. I. (2016). J. Phys.: Condens. Matter 28286001.

[8] Klein, Y. M. Kozłowski, M. Linden, A. Lacorre, Ph. Medarde, M. Gawryluk D. J. (unpublished). arXiv:2104.09873.

\section{Keywords: rare earth nickelates; single crystal; high-pressure; metal-to-insulator transition; antiferromagnetism}

This work was supported by the Swiss National Science Foundation through the NCCR MARVEL (Grant No. 51NF40-182892), and the R'equip Grant n. 461206021 139082. 